\title{
Contribuições epistemológicas para reduzir a distância teoria- prática da enfermagem na Colômbia
}

\author{
Contribuciones epistemológicas para disminuirla brecha teoría-práctica de \\ enfermeríaenColombia.
}

\section{Epistemological contributions to reduce the theory-practice nursing gap in Colombia}

\author{
William Iván López-Cárdenas* \\ Beatriz Helena Soto-Mora**
}

\section{Autor de correspondencia}

* Enfermero, Magister en Salud Pública. Profesor de la Facultad de Enfermería de la Universidad de Antioquia. Correo: wivan.lopez@udea.edu.co, orcid: https://orcid.org/0000-0001-9369-562X, Medellín, Colombia.

*** Enfermera, Magister en Salud Pública. Asesora de investigación, Facultad de Medicina, Universidad Nacional de Colombia. Correo: bhsotom@unal. edu.co, orcid: https://orcid.org/0000-0002-84112602, Bogotá, Colombia.
(C) Universidad Francisco de Paula Santander. Este es un artículo bajo la licencia CC BY (https://creativecommons.org/ licenses/by/4.0/) (c) (1)

\section{Resumo}

O desenvolvimento epistemológico de enfermagem tem atravessado diferentes fases nas que se tem definido o núcleo disciplinar o metaparadigma de enfermagem, a partir do qual tem se desenvolvido modelos conceituais e grandes teorias que têm definido a natureza, a missão e objetivos da disciplina, direcionando a pesquisa e a prática. Entretanto, seu nível de abstração conceitual limita o seu uso para a abordagem de fenômenos e aspectos próprios da prática de enfermagem. Ante esta situação, reflete-se sob as contribuições das teorias de rango médio, das teorias práticas ou de situação específica e da prática da enfermagem baseada na evidência, para a redução do distanciamento teoria-prática-pesquisa de enfermagem no contexto colombiano. Finalmente, propõe-se uma agenda de trabalho disciplinar e profissional que articule os desenvolvimentos teóricos e o conhecimento prático de enfermagem com as prioridades de saúde e cuidado na esfera nacional e global.

Palavras-chave: Teoria de enfermagem, modelos de enfermagem, pesquisa, enfermagem baseada em evidências.

\section{Resumen}

El desarrollo epistemológico de enfermería ha pasado por diferentes fases en las que se ha definido el núcleo disciplinar o metaparadigma de enfermería, a partir del cual se han desarrollado modelos conceptuales y grandes teorías que han definido la naturaleza, la misión y objetivos de la disciplina y han dado lineamientos generales para la orientación de la investigación y la práctica. Sin embargo, su nivel de abstracción conceptual limita su uso para el abordaje de fenómenos y cuestiones propias de la práctica de enfermería. Ante esta situación, se reflexiona sobre las contribuciones de las teorías de rango medio, de las teorías prácticas o de situación específica y de la práctica de enfermería basada en la evidencia, para la disminución de la brecha teoría-práctica-investigación de enfermería en el contexto colombiano. Finalmente, se propone una agenda de trabajo disciplinar y profesional que articule los desarrollos teóricos y el conocimiento práctico de enfermería con las prioridades de salud y de cuidado en la esfera nacional y global. 
Palabras clave: Teoría de enfermería, modelos de enfermería, investigación en enfermería, enfermería basada en la evidencia.

\begin{abstract}
The epistemological development of nursing has passed through different phases which have defined the disciplinary core or the metaparadigm of nursing, from which conceptual models and big theories have been developed and have defined the nature, mission and objectives of the discipline and also given general guidelines for its orientation towards research and practice. However, its level of conceptual abstraction limits its use for approaching phenomena and problems proper of the nursing practice. Facing this situation, a revision of the contributions of the theories of medium range, the practical theories for specific situations and the nursing practice based on evidence is made to reduce the theory-practice-research nursing gap in the Colombian context. Finally, a disciplinary and professional work agenda is proposed, which articulates the theoretical developments and the practical knowledge of nursing with the priorities of health and care in the national and global sphere.
\end{abstract}

Keywords: Nursing theory, nursing models, nursing research, evidence-based nursing.

\section{Introdução}

Todo conhecimento de enfermagem deveria estar orientado na solução de problemas que surgem do ato do cuidado, atendendo a sua natureza da disciplina prática (1). Partindo dessa afirmação entende-se que a prática de enfermagem é o senário onde emergem os questionamentos que devem ser resolvidos pelas pesquisas, e partindo da teorização desses achados, avançar na produção disciplinar que fundamenta a prática.

Entretanto, existe uma distância- amplamente documentada- entre a prática profissional e o desenvolvimento teórico investigativo da disciplina de enfermagem, particularmente os modelos conceptuais e as grandes teorias produzidas nas décadas de 70 y 80 do século $\mathrm{XX}$, onde o alto nível de abstração tem limitado a sua aplicação na prática profissional.

Cabe-se frisar que muitos fenómenos, problemas e questões de interesse surgem dos âmbitos clínicos ou comunitários onde atuam as enfermeiras são abordados sem ser utilizado o conhecimento disciplinar, de maneira semelhante, algumas das perguntas de pesquisa formuladas pelas teóricas e pesquisadoras da enfermagem têm contribuído de maneira limitada à inovação, fortalecimento ou solução de problemas da pratica profissional (2-6).

Neste sentido, não é alheia a prática da enfermagem colombiana, razão que gerou interesse na análise e reflexão do contexto da prática de enfermagem na Colômbia, identificando os desafios disciplinares, para reduzir a distância teoria-prática existente.

Para isso, faz-se necessário aproximar-se aos contextos históricos e as preocupações particulares que orientaram o trabalho das teóricas de enfermagem ao longo do século XX, podendo-se assim, compreender a estrutura do conhecimento disciplinar da enfermagem, as tensões e possibilidades de convergência com as problemáticas e assuntos de interesse da prática profissional, com o propósito de identificar alternativas que contribuam a reduzir o distanciamento referido.

Assim, a discussão estará orientada na abordagem do desafio que tem pela frente o desenvolvimento disciplinar da enfermagem no século XXI: o uso das teorias e enfoques mais próximos ao nível empírico como as teorias de rango médio, as de situações específicas e a prática baseada na evidência.

Como consequência, será refletido no primeiro momento, a trajetória e jerarquia do desenvolvimento disciplinar da enfermagem, as contribuições e limitações das teorias de rango médio, das teorias baseadas na prática e a prática da enfermagem baseada na evidência, para fortalecerem o vínculo entre a teoria e a prática da enfermagem. Finalmente, os elementos discutidos se presentam como uma agenda de desafios e projeções futuras da enfermagem colombiana em dois frentes de trabalho denominados: i) liderança e projeção social; ii) desenvolvimento disciplinar e geração de conhecimento.

\section{Desenvolvimento}

Trajetória do desenvolvimento disciplinar de enfermagem e contribuição ao fortalecimento do vínculo pesquisa-teoria-prática

Smith (1) refere que a formação inicial das enfermeiras do século XX fundamentou-se no paradigma das ciências 
ISSN-PRINT

1794-9831

E-ISSN 2322-7028

Vol. 17 No. 1

Ene - Abr 2020

Cúcuta, Colombia biomédicas, focando-se no desenvolvimento de habilidades para a aplicação do regime terapêutico prescrito pelo médico aos pacientes. Posteriormente, entre os anos 30 e 50, evidenciou-se o interesse pelo desenvolvimento de habilidades pedagógicas e administrativas das enfermeiras, pelo que os desenvolvimento teórico e investigativo dessa época ocupou-se em questões relativas ao papel da prática enfermeira e em menor proporção do desenvolvimento de conhecimento próprio da disciplina.

A partir da década de 50 surgiram questões e problemas de pesquisa relacionados com a disciplina da enfermagem, quando teoristas estadunidenses preocuparam-se pela definição do núcleo disciplinar da profissão, dando resposta a perguntas relativas à natureza, missão e objetivos da enfermagem, com o propósito de diferencia-la de outras ciências da saúde. Foi assim como estruturou-se o metaparadigma que direciona o núcleo disciplinar da enfermagem, conceituando o "cuidado da experiência e a vivencia da saúde humana" $(1,7)$.

A vigência deste paradigma têm sido objeto de discussões no final da segunda década de 2000, de cara ao confronto dos desafios do contexto social, tecnológico, ambiental e do atendimento em saúde do século XXI, cuja abordagem requer da redefinição do núcleo disciplinar da enfermagem, permitindo a incorporação de fenômenos como: a humanização , os significados e a experiência pessoal frente aos processos vitais, a autodeterminação nas práticas de saúde, o bem-estar, o relacionamento entre as pessoas, o meio-ambiente e a saúde $(8,9)$.

Partindo do marco de referência que determina a razão de ser da enfermagem iniciou-se uma etapa de identificação e conceptualização dos fenômenos de interesse para a prática profissional, pelo desenvolvimento de modelos conceituais, que de acordo com Roy (7), Fawcet\& de Santo (10), constituem o maior nível de abstração na jerarquia do conhecimento de enfermagem, dando a perspectiva u orientação geral para a prática. Por exemplo, o modelo de Roy e Orem, desenvolvem os conceitos metaparadigmáticos do cuidado, pessoa, entorno e enfermagem na perspectiva da adaptação e o autocuidado, respectivamente. $(7,10)$.

Deve-se apontar que a partir dos modelos conceituais têm se desenvolvido grandes teorias de enfermagem, que constituem o seguinte degrau na jerarquia do conhecimento proposta por Fawcet (7). As grandes teorias e os modelos conceituais constituem referentes fundamentais para a pesquisa e a prática. Estes referentes caracterizam- se por serem representações simbólicas y abstratas, cujo propósito é a descrição, a explicação ou a predição dos fenómenos de interesse para a enfermagem; entretanto, presentam dificuldades para operacionalizar seus conceitos além da sua comprobação empírica, pelo que sua capacidade de direcionar e orientar a prática de enfermagem é limitada $(1,7)$.

Referente a isto, a década de 1990 caracterizou-se por realizar importantes esforços na geração de teorias de menor nível de abstração conceitual, dirigidas a fenômenos específicos do cuidado, que fossem comprováveis e pudessem orientar e prescrever a prática enfermeira. Assim, surgiram as teorias focadas em aspetos concretos como: o manejo de sintomas desagradáveis, o autocuidado, a transição à maternidade, a auto-eficácia, o autocuidado na doença crônica, a carga do cuidado e a incerteza; proposições que tem permitido o maior nível de articulação da teoria e a prática da enfermagem e que têm sido denominadas teorias de rangomédio $(11,12)$.

As teorias d enfermagem de rango médio caracterizam-se por terem um limitado número de conceitos claramente definidos, descrevendo um fenómeno específico da prática de enfermagem como: o autocuidado, a adaptação , o sofrimento, a percepção da dor, etc. Estes fenômenos podem- ser abordados desde diferentes senários de cuidado como são o contexto hospitalar, o lar o a comunidade. É uma realidade que o seu nível de abstração não permite a prescrição da prática de enfermagem, seus conceitos são de fácil operacionalização, permitindo a orientação da prática e o desenvolvimento de ferramentas de medição para a comprovação teórica (11-13).

Finalmente, na jerarquia do conhecimento de enfermagem encontrasse um nível de teoria com menor abstração, com capacidade de prescrever sua prática e a sua comprobação teórica, denominadas como microteorias, teorias de situação específica ou teorias práticas, que representam o degrau mais próximos à prática profissional na jerarquia do conhecimento disciplinar da enfermagem (14).

Cabe-se frisar que estas teorias surgem e incorporam elementos do contexto onde se dá o ato do cuidado de enfermagem, pelo que têm um número limitado de conceitos com alcance limitado e específico; desta maneira são capazes de prescrever ações específicas de cuidado facilmente medíveis , permitindo a comprobação teórica (14). Como exemplos podem ser citas as teorias sobre: os comportamentos em saúde de mulheres estaduniden- 
ses de origem coreana com hepatites $\mathrm{B}$, autocuidado em pacientes com insuficiência cardíaca, contribuições dos cuidadores ao autocuidado na insuficiência cardíaca, a adaptação das mulheres grávidas de alto risco e a abordagem de crises de emergência de pessoas com doenças mentais graves e persistentes, entre outras (15-19).

Entre as vantagens das teorias de rango médio encontrase a sua capacidade da abordagem do mesmo fenômeno desde diferentes senários onde se desenvolve a prática de enfermagem. Por outro lado, a principal vantagem das teorias de situações específicas é a sua capacidade de prescriçãoda prática e a comprobação teórica, embora não seja possível a sua utilização em outros contextos. A principal crítica dos opositores das teorias práticas radica em considera-las procedimentos que carecem de baseamento num adequado conjunto de conhecimentos da ciência de enfermagem $(12,14)$.

Sobre isso, Im (1) tem discutido as possibilidades de construção de teorias de rango médio e de situação específica, traçando caminhos metodológicos de caráter indutivo ou dedutivo (11). Em concordância, existe a tendência atual em desenvolver teorias de situações específicas, ancoradas em teorias de rango médio, o que permite maior relação entre os diferentes níveis da jerarquia de teorias da enfermagem, além do fortalecimento do vínculo entre a teoria e a prática. Assim, por exemplo, a teoria das Transições de Meleis (20), têm surgido múltiplas teorias de situações específicas que enfocam-se em: as transições à maternidade de neonatos internados nas unidades de terapia intensiva, o climatério em mulheres migrantes coreanas, o cuidado transicional em adultos internados com insuficiência cardíaca ou a sexualidade no idoso, entre outras (20).

\section{Contribuições e limitantes da prática de enfermagem baseada na evidência para a redução do distancia- mento existente}

Cochrane (1972) utilizou por primeira vez o termo Prática Baseada na Evidência (PBE) para referir-se à necessidade de fundamentar as decisões médicas que surgem dos estudos clínicos aleatorizados. Posteriormente, Sackertt (1996) introduziu o conceito Medicina Baseada na Evidência (MBE) para referir-se à integraçãoda experiência clínica, a evidência disponível, os valores e as preferências do paciente para tomar decisões clínicas. Entretanto, suas origens remontam-se à disciplina enfermeira com a publicação das notas de enfermagem de Florence Nightingale (1859), onde fazia referência à necessidade de melhorar os resultados nos pacientes a partir de evidências sólidas. Neste sentido, Nigthingale demostrou diferenças na limpeza das mãos com o uso exclusivo da água comparado com o uso de sabonete $(21,22)$.

O conceito de Enfermagem Baseada na Evidência (EBE), introduziu-se ao final dos 90 como adaptação do conceito da disciplina médica. Definiu-se com o fim de resolver problemas sobre a toma de decisões clínicas, num contexto de cuidado, partindo da integração da melhor evidência disponível, a experiência clínica, as necessidades e preferencias do paciente, com o propósito de fornecer assistência de qualidade, segura e custo-efetiva $(22,23)$.

Cabe-se frisar que a maior contribuição da EBE para a redução do distanciamentos teoria-prática da enfermagem radica no seu potencial para transferir e aplicar à prática conhecimentos desenvolvidos com pesquisa. Entretanto, dentro das suas limitações evidenciam-se dois aspectos que são problemáticos: a natureza ontológica do conceito de evidência e as possibilidades de integração da mesma com as habilidades e experiência da enfermeira e as preferencias do paciente.

Relacionado com a primeira problemática, a PBE fundamenta-se no principio de racionalidade técnica, conceito que surgiu da teoria da administração científica de Taylor, que propôs a fragmentação e distribuição dos processos produtivos com a finalidade de administrar a força do trabalho e incrementar a produtividade da indústria. No contexto hospitalar, a racionalidade técnica expressase no principio de custo-efetividade das intervenções e na necessidade de predizer e controlar a prática clínica mediante padronização (24).

Dito enfoque lógico-positivista privilegia a evidência que proveem de modelos empíricos analíticos e considera o atendimento sanitário como um processo estável, regular, independente do contexto, previsível, quantificável e objetivável; ao mesmo tempo, desconhece a natureza ontológica dos saberes da disciplina de enfermagem relacionados com a interação humana, que configuram o ato do cuidado, processo subjetivo, singular, instável, ambíguo e imprevisível $(24,25)$.

Desde a perspectiva da racionalidade técnica, o cuidado de enfermagem, é reduzido à aplicação de uma receita de intervenções padronizadas que desconsideram o juízo clínico da enfermeira, que está fundamentado no conhecimento do paciente, em múltiplas teorias sobre enfermagem e no seu conhecimento prático. Autoras como Norlyk e Col (24) têm nomeado esta situação como a 
ISSN-PRINT

1794-9831

E-ISSN 2322-7028

Vol. 17 No. 1

Ene - Abr 2020

Cúcuta, Colombia "macdonalização da enfermagem", termo que pretende exaltar a excessiva padronização e consequente desumanização do cuidado de enfermagem (24).

Neste respeito, empregando um estudo qualitativo-narrativo que avalio a implementação de um programa de EBE sobre o egresso precoce após artroplastia de pelve, Norlyk e Col (24) demostraram que a principal preocupação da enfermeira foi executar a guia de prática clínica para a promoção da autonomia do paciente, conseguir a sua mobilidade precoce e o egresso hospitalar ao terceiro dia. Na narrativa não foi evidenciado o esforço da enfermeira pela compreensão da situação particular de cada paciente, por necessidades fundamentais do cuidado como a dor, as náuseas, a incerteza para desenvolver as atividades do dia a dia e a preocupação com a transição da sua recuperação no seu lar, não foram consideradas dentro do plano de cuidados. Desde a perspectiva da estandardização, a paciente conseguiu o egresso precoce ao terceiro dia do procedimento cirúrgico; entretanto, da perspectiva do cuidado, a paciente experimentou distanciamento, incompreensão por parte da enfermeira, quem não teve sensibilidade para identificar as suas necessidades de cuidado (24).

Deve-se destacar que as críticas para a EBE apontam que a evidência gerada, na maioria dos casos inserida nos procedimentos do âmbito clínico, fazem poucas contribuições ao desenvolvimento de novos conhecimentos na enfermagem (24). Resolver essas tensões requerem de uma análise crítica e da redefinição do conceito de evidência na disciplina da enfermagem, propondo o enfoque holístico que contenha os diversos padrões e manifestações do conhecimento teórico e prático da enfermagem, de maneira que transitem em direção da prática baseada no conhecimento e do saber enfermeiro. Igualmente, requer do fortalecimento e da ética do cuidado, procurando uma relação empática e o esforço constante para compreender a situação do paciente, de maneira que o ato do cuidado seja realizado no contexto singular, evitando a "coisificação" do ser humano como uma patologia ou conjunto de tarefas e atividades a serem realizadas (24).

Respeito à segunda problemática da EBE, a integração tem sido pouco desenvolvida e compreendida como conceito, devido à maioria de pesquisas e programas de treinamento que têm sido formulados fortalecendo habilidades para a formulação de preguntas clínicas, a procura e utilização de evidência científica e em menor medida, em compreender como deveria dar-se a integração entre evidência, experiência e as preferências do paciente
$(23,24)$. Em algumas ocasiões predomina o uso da evidência científica e em outros, a participação do paciente limita-se à sua opinião sobre padrões previamente estabelecidos. Frente a isto, a emergência de conceitos como participação do paciente, atenção centrada no paciente e toma de decisões compartilhadas, tem contribuído ao posicionamento do paciente como sujeito ativo e protagonista do ato do cuidado (24).

Promover a integração entre os componentes da EBE requer transformações curriculares na formação profissional, que prejudiquem o impacto da racionalidade técnica sobre o processo formativo, atendendo a fragmentação do conhecimento e privilegiar o treinamento de habilidades que devem dominar-se para o exercício profissional, concebendo a prática de enfermagem como uma prática instrumental, tal como afirma Jose Luis Medina (25). Autoras como Benner e Schön tem realizado contribuições para superar esta limitação por meio do desenvolvimento da noção do conhecimento prático-reflexivo, que constitui-se desde os significados que surgem da interação sujeito-sujeito durante o ato do cuidado. Partindo desta perspectiva, concebe-se que a prática profissional devese desenvolver em contextos dinâmicos que presentam problemas ambíguos e não previsíveis , cuja abordagem e solução requer do exercício reflexivo paralelo e à ação, que permita a geração, a formulação e validação de hipóteses de compreensão aplicadas a situações de cuidado únicas e singulares; é dizer, o juízo clínico e a toma de decisões devem ser produto da articulação entre o saber disciplinar, o contexto do cuidado e a comunicação com o paciente, que em conjunto, constituem o saber prático da enfermeira (25).

Embora as limitações e problemáticas previamente descritas, deve-se considerar que a EBE abre a possibilidade de gerar evidência sobre o cuidado de enfermagem na prática, de responder a perguntas de pesquisa específicas do âmbito clínico, assim como avaliar e promover a prática de enfermagem socialmente responsável (4). Neste sentido, é imprescindível trabalhar na transformação dos aspectos que limitam as contribuições da EBE no desenvolvimento disciplinar de enfermagem. Uma porta de saída para esta situação, é a incorporação da EBE na graduação, orientada na perspectiva crítica fundamentada no conhecimento de enfermagem (26) ou no desenvolvimento de teorias sobre enfermagem que incorporem esta tecnologia de gerência do cuidado, como a teoria de rango médio de Cheng e Col (27), nomeada de "criar raízes" (taking root) focada no processo de implementação da EBE em China $(26,27)$. 
Contexto e desafios para o fortalecimento do vínculo entre o desenvolvimento teórico e a prática de enfermagem na Colômbia

A prática de enfermagem na Colômbia desenvolve-se num contexto complexo que contribui na ampliação do distanciamento entre a teoria e a prática existente. No primeiro lugar, a prestação dos serviços de saúde na Colômbia estrutura-se baseado num modelo de mercado orientado por valores como o lucro, a eficiência económica e o custo-efetividade, que têm contribuído na desumanização do cuidado da saúde das pessoas (28).

No segundo lugar, as instituições hospitalares privilegiam o regime de tratamento médico, razão pela qual as atividades de outros integrantes da equipe de saúde devem orientar-se em função do cumprimento do regime terapêutico estabelecido pelo médico.

Isto se traduz na mandato de um excessivo volume de pacientes aos enfermeiros, obrigando-os a delegar as ações de cuidado ao pessoal técnico. A prática ambulatória mostrou um padrão semelhante, com tempos para $\mathrm{o}$ atendimento de enfermagem de 20 minutos, que predominantemente dedicam-se ao preenchimento de formatos e registros exigidos para a faturação, cobro e registro das atenções em saúde.

Além disso, o gerenciamento que caracteriza o atendimento hospitalar do enfermeiro, o obriga a que o seu tempo seja predominantemente dedicado a atividades administrativas como solicitação de leitos, pedidos de alimentação, medicamentos e insumos. Em todos os casos, o tempo para estabelecer uma relação do cuidado com os pacientes es limitado.

Outro aspecto contextual fundamental faz referência à ausência de sistematização dos prontuários e continuidade na pesquisa de enfermagem nas instituições hospitalares da Colômbia, fazendo que a produção de conhecimentos seja exclusiva das instituições de ensino. Por tudo isto o contexto da prática configura-se como um território infértil para a geração de evidência de enfermagem que direcione a prática e a produção teórica.

Por outro lado, a trajetória do desenvolvimento disciplinar da enfermagem na Colômbia evidencia um campo jovem e em construção. Os primeiros programas de mestrado em enfermagem de América Latina surgiram na Colômbia e na Venezuela em 1969 e os primeiros cursos de doutorado deram-se no Brasil (1981), Venezuela (1999), México (2003) e finalmente na Colômbia em
2004 e 2009 na Universidade Nacional da Colômbia e na Universidade de Antioquia (29). O anterior permite concluir que a enfermagem na Colômbia têm estado direcionada pelas teorias formuladas pelas norte-americanas.

Sem dúvida nenhuma, um dos principais desafios disciplinares da enfermagem colombiana é produzir conhecimento localmente situado, que dê conta das experiências e vivencias da saúde da população colombiana que respondaàs necessidades particulares do contexto onde desenvolve a sua prática. $\mathrm{O}$ anterior não significa afastarse do desenvolvimento teórico das enfermeiras norteamericanas, de fato, a sua maior contribuição têm sido a definição dos conceitos que conformam o metaparadigma da enfermagem e outros que constituem fenômenos de interesse para a prática como a adaptação, conforto, bem-estar, afrontamento, angustia, sofrimento, etc (30).

Os desafios atuais da enfermagem em Colômbia podem descrever-se, sugerindo uma agenda que deverá atender dois frentes denominados: i) liderança e projeção social; e ii) desenvolvimento disciplinar e geração do conhecimento.

Relacionado com a liderança e a projeção social, a enfermeira colombiana afronta desafios como: contribuir à construção de uma sociedade justa, equitativa e em paz; a formulação de teorias e modelos de cuidado para dar resposta a prioridades de saúde no nível nacional, regional e local, com alto nível de suporte clínico e empírico; e, fomentar o trabalho colaborativo com outras profissões para o desenvolvimento de teorias de cuidado da saúde (31). Neste sentido, Marlaine (1), Roy (7) e Willis (8), apontam que o aumento da esperança de vida exigirá respostas da enfermeira frente a necessidade de cuidados de longa duração, que privilegiarão às comunidades e os lares como senários ideais para a prática.

Deverá considerar-se também, que a urbanização e os fenômenos migratórios configuraram novos senários de cuidado urbano, caracterizados por mudanças nas condições de vida, do meio ambiente, a segurança, os estilos de vida e as necessidades em saúde. De igual maneira, as transformações tecnológicas para a atenção em saúde têm permitido o desenvolvimento de novas modalidades de atendimento como a telessaúde, que exigirá a reformulação dos conceitos e as formas de relação com os pacientes $(1,7,8)$.

Para dar resposta aos novos desafios são propostas ações na área da promoção da saúde mental como: recuperação da memoria do conflito, acompanhamento na transição à 
ISSN-PRINT

1794-9831

E-ISSN 2322-7028

Vol. 17 No. 1

Ene - Abr 2020

Cúcuta, Colombia vida social de ex-combatentes, desenvolvimento de projetos comunitários que fortaleçam os grupos sociais, a resiliência, a reconciliação a redução de danos de consumo problemático de substancias psicoativas, a promoção da saúde mental e, contextos de conflito armado urbano e desenvolver capacidades para a identificação e intervenção de problemas de saúde mental como o suicídio e a depressão.

Outra linha de trabalho faz referencia à incorporação de aspectos subjetivos, culturais e de contexto sóciohistórico, para conseguir a compreensão das práticas de autocuidado e de risco ao redor do processo saúdedoença-cuidado-atenção-morte; citando como exemplos a mutilação genital em mulheres de comunidades indígenas, a prevenção de infecções de transmissão sexual em sociedades machistas que vivem em poligamia, ou a adoção de condutas sexuais de risco em populações de homens que que tem relações sexuais com outros homens.

De igual maneira, deve-se privilegiar a articulação interdisciplinar com as ciências sociais, a arte e o saber popular, para a construção de novas linguagens do cuidado. Como condição para esta construção dialética, surge a necessidade da des-aprendizagem da enfermeira para abrir campo a emergência de "outras" possibilidades de ser, pensar, relacionar-se, cuidar-se e cuidar os outros.

Igualmente, deve-se propiciar a promoção e consolidação das redes sociais para o cuidado da saúde baseados na comunidade e desenvolvimento de modelos de cuidado para pessoas em situação de vulnerabilidade tais como: pessoas com deficiência física, habitantes de rua, pessoas na cadeia, mulheres transgênerono exercício da prostituição, etc.

Do mesmo modo, requer fomentar-se a construção coletiva de programas de saúde pública com perspectiva cultural, o desenvolvimento de políticas públicas para a promoção da saúde; assim como a apropriação de saberes interdisciplinares para a gestão territorial da saúde e da enfermagem.

Também é necessário implementar estudos orientados à economia e carga de cuidado de pessoas com doenças crônicas; assim como o desenvolvimento de modelos para o cuidado no lar, que permitam gerar capacidades para prestar cuidados de longa duração.

Do mesmo modo, podem-se desenvolver programas de formação como residências em enfermagem, que permi- tam através da formação em serviço o aprofundamento e especialização na prática avançada em campos como: atenção primária à saúde, atenção do parto, cuidado familiar e de pessoas com doenças crônicas; os mesmo gerariam maior nível de competências para o desenvolvimento de atividades como a prescrição de medicamentos na atenção primária à saúde ambulatorial, por exemplo.

Por outro lado, dar maior visibilidade à enfermagem na estrutura administrativa das instituições de saúde, criando direções de enfermagem. Portanto, o nível hospitalar deve retomar-se o cuidado direto pelo profissional com apoio do pessoal técnico afim de facilitar ações de conforto. $\mathrm{O}$ anterior deve estar respaldado pela atualização da lei 266/1996 e o código deontológico da enfermagem, com o objetivo de estabelecer a regulação do perfil do técnico auxiliar de enfermagem, assim como as diferencias nas competências com a formação profissional.

De forma semelhante, convém fomentar experiências que promovam a construção de alianças entre colegas e colaboração entre alunos, professores e professionais.

Como ação de visibilidade é proposta a participação da campanha mundial "NursingNow", liderada pelo Conselho Internacional de Enfermagem e Organização Mundial da Saúde para reconhecer e fortalecer o papel da enfermeira dentro dos sistemas de saúde.

Neste sentido,"NursingNowAntioquia" pretende realizar aportes mediante o desenvolvimento de pesquisas colaborativas entre hospitais e universidades, pretendendo validar as práticas que gerem insumos para a toma de decisões em matéria de saúde.

Finalmente, destaca-se o trabalho gremial como área de consolidação da disciplina e da profissão. Neste ponto, o Conselho Técnico Nacional de Enfermagem liderará a construção participativa do Plano Nacional de Enfermagem 2020-2030, documento referencia para a toma de decisões em política pública relacionadas com condições laborais, formação e reconhecimento social da profissão.

No segundo frente de trabalho: o desenvolvimento disciplinar e geração de conhecimento, a enfermagem reque de desenvolvimento de evidências que justifiquem mudanças no gerenciamento e delegação do ato do cuidado, com a finalidade de reduzir as consequências associadas com deficiente qualidade do cuidado como: mortalidade, quedas, infecções hospitalares, entre outros eventos adversos. 
Para isso, requer de desenvolvimento de novos papeis da enfermeira no atendimento clínico, os quais concretam-se na criação de unidades de pesquisa lideradas por doutores em enfermagem (32); igualmente, deve-se promover a pesquisa sobre: o cuidado direto profissional, a definição de padrões para determinar o número de pacientes assignados para cada enfermeiras nos diferentes serviços, o custo das diferentes intervenções da enfermagem; assim como o desenvolvimento e validação de modelos de cuidado que incorporem a taxonomia da enfermagem na atenção clínica e comunitária.

Também é necessária a incorporação da epistemologia do cuidado de enfermagem nas políticas atuais do sistema de saúde, como exemplo é proposto o aproveitamento das oportunidades de consulta familiar, do adolescente, $\mathrm{o}$ assessoramento no aleitamento materno que surgem das Guias Integrais de Atendimento à Saúde . Neste sentido, podem-se aplicar modelos teóricos orientadores de cuidado, como a teoria de rango médio das transições de Afaf Meleis, que servem para situações vitais e sociais relacionadas com o envelhecimento, a criança dos filhos ou a doção do rol materno ou paterno.

É importante considerar que o conhecimento emergente é fundamental para a criação de teorias de rango médio, dirigidas à compreensão dos fenómenos locais e situações como: a promoção da saúde mental em contextos de violência urbana; o cuidado da população migrante; a ablação feminina ; o cuidado em comunidades negras, indígenas e rurais em contextos de isolamento geográfico.

Portanto, deve-se promover a criação de uma rede de inovação social para o cuidado da saúde, que permitam o desenvolvimento de projetos multicêntricos no país para a abordagem de prioridades de cuidado que tenham a ver com: a saúde materna, a infantil, o envelhecimento e as doenças crônicas. De forma paralela, tem que ser implementada a geração de alianças entre universidades, o setor público e privado para o desenvolvimento de projetos Spin Off, que permitam a aplicação do conhecimento de enfermagem na solução de problemas práticos relacionados com a atenção em saúde de indivíduos e coletivos.

É conveniente incentivar a criação de um banco de pesquisa de enfermagem de acesso livre, com sistematização de experiências exitosas em enfermagem para a aplicação do aprendizagem obtido em contextos semelhantes.
Propõe-se também a criação do observatório de prática da enfermagem, onde poderão se realizar ações como a monitorização constante de condições educativas e laborais do recurso humano em enfermagem; a aplicação de uma escala quinquenal de caracterização do recurso humano a nível nacional, como insumo para a construção de uma linha de referência para o desenvolvimento de políticas públicas em enfermagem que inclua aspectos como condições laborais, formação, legislação, etc.

Por último, deve-se destacar que a globalização e a revolução tecnológica presentam desafios relacionados com: a conformação de redes de conhecimento em enfermagem que permitam o intercâmbio de conhecimentos e experiências, a mobilidade de alunos da graduação em enfermagem em diferentes níveis de sua formação, a pesquisa colaborativa e multicêntrica e a identificação de competências genéricas dos profissionais que favoreçam sua migração com fins laborais. Todos estes aspectos estarão orientados à construção do marco de referencia global para a pesquisa, a teoria e a prática de enfermagem $(7,31,33)$.

\section{Conclusões}

- A enfermagem é uma disciplina prática com desenvolvimentos teóricos recentes, que no começo orientaram-se na definição dos conceitos que configuram o núcleo disciplinar, assim como das diretrizes gerais para orientar a pesquisa e a prática de enfermagem. Partindo da década de 1990 têm se evidenciado maior interesse pelo relacionado com fenômenos específicos da prática de enfermagem como a vivencia das experiências da saúde, doença e a morte das pessoas, permitindo uma maior aproximação entre a teoria e a prática da enfermagem, através do desenvolvimento de teorias de rango médio e teorias de situação específica, caracterizadas pelo seu limitado número de conceitos e a possibilidade de executá-los na prática,. Entretanto, o desenvolvimento de teorias prescritivas é recente, pelo que ainda se mantem o distanciamento entre a teoria e a prática.

- A prática de enfermagem baseada na evidência têm o potencial de gerar conhecimento em enfermagem orientado a resolver assuntos inerentes à prática, sempre que o conceito de evidência se redefina à partir da filosofia de enfermagem e se apliquem estratégias que permitam o desenvolvimento do pensamento reflexivo que oriente a prática de enfermagem, de forma que o juízo 
ISSN-PRINT

1794-9831

E-ISSN 2322-7028

Vol. 17 No. 1

Ene - Abr 2020

Cúcuta, Colombia clínico da enfermeira integre o seu conhecimento disciplinar, assim como as necessidades do paciente e do contexto da situação do cuidado (26)

- A redução e fechamento do distanciamento entre a teoria e prática de enfermagem no contexto colombiano representa um desafio para o desenvolvimento dessa disciplina para o século XXI, sendo uma condição indispensável para responder aos reptos disciplinares e profissionais que impõem fenômenos como: a transição demográfica, a epidemiológica, a urbanização, as inequidades e a interculturalidade, a projeção social da profissão o desenvolvimento disciplinar, a transformação do contexto para a prática e a conformação de comunidades de conhecimento.

\section{Conflito de interesses}

Os pesquisadores declaram não ter conflito de interesses.

\section{Referências Bibliográficas}

1. Smith M. RegeneratingNursing'sDisciplinaryPerspective. Advances in NursingScience. [Internet]. 2019[consultado 20 de septiembre de 2019]; 42(1):3-16. Disponible en: https://dialnet.unirioja.es/ servlet/articulo? codigo $=6848120$

2. Gomes BM, Santos de Andrade MJ, Pinto PM, Pitta LR, Caniçali PC. Reflexões teóricas e metodológicas para a construção de teorias de médio alcance de enfermagem. Texto Contexto Enferm[Intern et].2017[consultado03deseptiembrede2018]; 26(4):1-8.Disponible en: http://www.scielo.br/pdf/tce/ v26n4/0104-0707-tce-26-04-e1420017.pdf

3. Mccrae N. WhitherNursingModels? The valueofnursingtheory in thecontextofevidence-basedpracticeandmultidisciplinaryhealthcare. J AdvNurs. 2012; 68(1): 222-229.

4. Ellis P. Whatisevidence-basednursing?. En: Ellis Peter and Standing Mooi. Evidence-base practice in nursing. 4 Ed. London: LearningMatters; 2019.

5. Leach M, Tucker B. Currentunderstandings of theresearch-practice gap in nursing: A mixed-methodsstudy. Collegian [Internet]. 2018[consultado 20 de septiembre de 2019]; 25(2):171-179. Disponible en: https://www.sciencedirect.com/science/article/abs/pii/S1322769617300938

6. Bond AE, Eshah NF, KhaledMB, Hammad AO, Habashneh S, et al. Who uses nursingtheory? A univariatedescriptiveanalysis of fiveyears' researcharticles. Scand J CaringSci [Internet]. 2011[consultado10deseptiembrede2018]; 25(2):404-409. Disponible en: https://www.ncbi.nlm.nih.gov/ pubmed/20950408

7. Roy C. NursingKnowledge in the 21st Century Domain-Derived and Basic SciencePractice-Shaped. Advances in NursingScience [Internet]. 2019 [consultado 29 de septiembre de 2019]; 42(1):28-42. Disponible en: https://insights.ovid.com/crossref?an=00012272-201901000-00005

8. Willis D, Grace P, Roy C. A Central UnifyingFocusforthe Discipline FacilitatingHumanization, Meaning, Choice, Quality of Life, and Healing in Living and Dying. Advances in NursingScience. [Internet]. 2018 [consultado 20 de septiembre de 2019]; 31(1):28-40. Disponible en: https://insights.ovid. $\underline{\text { com/article/00012272-200801000-00012 }}$

9. Roy C. Key Issues in NursingTheoryDevelopments, Challenges, and FutureDirections, NursingResearch. [Internet]. 2018 [consultado 17 de septiembre de 2019]; 67(2):81-92. Disponible en: https:/insights.ovid.com/crossref?an=00006199-201803000-00005

10. Fawcett J, DeSanto MS. Thestructure of contemporarynursingknowledge. En: Fawcett J, DeSanto MS.Contemporarynursingknowledge: Analysis and evaluation of nursingmodels and theories. 3 ed. Philadelphia: FA Davis Company; 2013. Pp 3-25.

11. Im E. TheoryDevelopmentStrategies for Middle-Range Theories. Advances in NursingScience. [Internet]. 2018 [consultado 20 de septiembre de 2019];41(3):275-292. Disponible en: https://journals.lww. com/advancesinnursingscience/Abstract/2018/07000/Theory_Development_Strategies_for_Middle $\underline{\text { Range.8.aspx }}$ 
12. Liehr P, Smith MJ. Middle Range Theory A Perspective onDevelopmentand Use. Advances in NursingScience. [Internet]. 2017 [consultado 20 de septiembre de 2019]; 40(1):51-63. Disponible: https:// journals.lww.com/advancesinnursingscience/Abstract/2017/01000/Middle_Range Theory_A_Perspective on Development.6.aspx

13. Smith MJ, Liehr P. Middle Range Theory for Nursing. 4 ed. New York: Springer Publishing Company; 2018.

14. Im E. Situation-SpecificTheoriesFromtheMiddle-Range TransitionsTheory. Advances in NursingScience. [Internet]. 2018 [consultado 20 de septiembre de 2019]; 37(1):19-31. Disponible en:https:// journals.lww.com/advancesinnursingscience/Abstract/2014/01000/Situation_Specific_Theories From the Middle Range.5.aspx

15. Lee H, Fawcett J, Hyang Yang JH, Hann HW. Correlates of Hepatitis B Virus Health RelatedBehaviors of KoreanAmericans: A SituationSpecificNursingTheory. J NursScholarsh. [Internet]. 2012[consultado13deseptiembrede2018]; 44(4):315-22. Disponible en: https://sigmapubs.onlinelibrary.wiley. com/doi/abs/10.1111/j.1547-5069.2012.01468.x

16. Herber O, Kastaun S, Wilm S, Barroso J. FromQualitative Meta-SummarytoQualitative Meta-Synthesis: Introducing a New Situation-SpecificTheoryofBarriersandFacilitators for Self-Care in PatientsWith Heart Failure. QualitativeHealthResearch[Internet]. 2018 [consultado 20 de septiembre de 2019]; 29(1):96-106. Disponible en: https://journals.sagepub.com/doi/abs/10.1177/1049732318800290

17. Fawcett J, Tulman L. Adaptation to High-RiskChildbearing: A PreliminarySituation-SpecificTheory. Aquichán [Internet]. 2018 [consultado 18 de septiembre de 2019]; 18(4):407-414. Disponible en: http://www.scielo.org.co/pdf/aqui/v18n4/1657-5997-aqui-18-04-00407.pdf

18. Vellone E, Riegel B, Rosaria A. A Situation-SpecificTheoryofCaregiverContributionsto Heart Failure Self-care. Journal of Cardiovascular Nursing. [Internet]. 2019 [consultado 18 de septiembre de 2019]; 34(2):166-173. Disponible en: https://journals.lww.com/jcnjournal/Abstract/2019/03000/A_Situation_Specific Theory_of_Caregiver.12.aspx

19. Brennaman L. CrisisEmergencies for IndividualswithSevere, Persistent Mental Illnesses: A SituationSpecificTheory. ArchPsychiatrNurs[Internet]. 2012 [consultado21deseptiembrede2018]; 26 (4):25160. Disponible en: https://www.sciencedirect.com/science/article/pii/S0883941711001579.

20. Meleis, Afaf I. TransitionsTheory. En: NursingTheories\&NursingPractice. 4 ed. Philadelphia: Davis Company; 2015.Pp 361-380

21. Williams B, Perillo S, Brown T. What are thefactors of organisational culture in healthcaresettingsthatact as barriers to theimplementation of evidence-basedpractice?. Nurse EducToday[Internet]. 2015[consultado21deseptiembrede2018]; 35(2):e34-e41. Disponible en: https://www.sciencedirect. com/science/article/pii/S0260691714003815

22. Mackey A, Bassendowski S. Thehistory of evidence-basedpractice in nursingeducation and practice. J ProfNurs[Internet]. 2017[consultado02octubrede2018]; 33(1):51-55. Disponible en: https://www.researchgate.net/publication/303358913 The History_of_Evidence_Based_Practice in_Nursing_Education and Practice

23. 23. Taylor M, Priefer BA, AltWhite AC. Evidence-basedpractice:Embracingintegration. Nurs Outlook. [Internet]. 2017 [consultado 30 deseptiembrede2018]; 64(6): 575-582. Disponible en: https:// www.sciencedirect.com/science/article/abs/pii/S0029655416300513

24. Norlyk A, Haahr A, Dreyer P, Martinsen B. Lost in transformation? Revivingethics of care in hospital cultures of evidence-basedhealthcare. NursInq. 2017; 24(3): 1-7.

25. Medina JL. La enseñanza reflexiva de profesionales de la enfermería. De los reduccionismos pedagógicos a los enfoques reflexivos de la complejidad. En: Dossier presentado al taller de capacitación para docentes en transformación curricular de la Facultad de Enfermería-Universidad de Antioquia. Medellín: Universidad de Antioquia; 2018. 
ISSN-PRINT

$1794-9831$

E-ISSN 2322-7028

Vol. 17 No. 1

Ene - Abr 2020

Cúcuta, Colombia
26. Cui C, Li Y, Geng D, Zhang H, Jin C. Theeffectiveness of evidence-basednursingondevelopment of nursingstudents' criticalthinking: A meta-analysis. Nurse EducationToday. 2018; 65:46-53.

27. Cheng L, Broome ME, Feng S, Hu Y. TakingRoot: a groundedtheoryonevidence-basednursingimplementation in China. IntNursRev [Internet]. 2018 [consultado29deseptiembrede2018];65(2):270-278. Disponible en: https://onlinelibrary.wiley.com/doi/10.1111/inr.12396

28. López WI, Pereira MA, Machado CV. Trajetória das relações público-privadas no Sistema de Saúde da Colômbia de 1991 a 2015. Cad. Saúde Pública [Internet]. 2017 [consultado16deseptiembrede2018]; 33(2):1-16. Disponible en: http://www.scielo.br/scielo.php?script=sci arttext\&pid=S0102$\underline{311 X 2017001405003}$

29. Da Silva R. Pós-graduação e a pesquisa em enfermagem na américa latina: avanços e desafios.RevCuid [Internet]. 2015 [consultado16deseptiembrede2018]; 6(2):1019-21. Disponible en: https://revistacuidarte.udes.edu.co/index.php/cuidarte/article/view/307

30. Fawcett J. Tendencias de investigación en enfermería. Aquichán [Internet]. 2014 [consultado26deseptiembrede2018]; 14(3):289-293.Disponible en: http://aquichan.unisabana.edu.co/index.php/aquichan/ article/view/4695

31. Meleis A. Mi deseo para una Agenda de Investigación Global en Enfermería. Rev. Latino-Am. Enfermagem [Internet]. 2015 [consultado30deseptiembrede2018]; 23(4):569-70. Disponible en: http:// www.scielo.br/pdf/rlae/v23n4/es_0104-1169-rlae-23-04-00569.pdf

32. BrantJ. BridgingtheResearch-to-Practice Gap: The Role of the Nurse Scientist. SeminOncolNurs [Internet]. 2015 [consultado24deseptiembrede2018]; 31(4):298-305. Disponible en: https://www.sciencedirect.com/science/article/pii/S0749208115000662

33. Holland S, Magama M. Evidence based practice translated through global nurse partnerships. Nurse EducPract [Internet]. 2017[consultado23deseptiembrede2018]; 22:80-82. Disponible en: https://www. sciencedirect.com/science/article/pii/S1471595316302670 